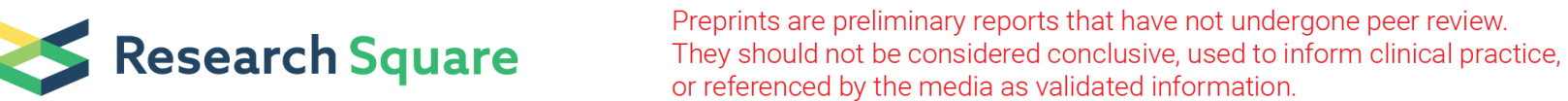

\section{Zhizhu Kuanzhong Capsule in Treating Patients With Functional Dyspepsia Postprandial Distress Syndrome: Study Protocol For a Multicenter, Randomized, Double-blind, Placebo-Controlled, Parallel Group Clinical Trial}

\section{Mengli Xiao}

China Academy of Chinese Medical Sciences

Linda LD Zhong ( $\triangle$ ldzhong@hkbu.edu.hk)

Hong Kong Baptist University https://orcid.org/0000-0002-3877-1914

Wai Ching Lam

Hong Kong Baptist University

Yingpan Zhao

China Academy of Chinese Medical Sciences Xiyuan Hospital

Kok-Ann Gwee

National University of Singapore Faculty of Medicine: National University Singapore Yong Loo Lin School of Medicine

Gerald Holtmann

Princess Alexandra Hospital

Jan Tack

University Hospitals Leuven

Hidekazu Suzuki

Tokai University

Min-Hu Chen

Sun Yat-sen University First Affiliated Hospital

Yinglian Xiao

Sun Yat-sen University First Affiliated Hospital

Xiaohua Hou

Huazhong University of Science and Technology

Jinsong Liu

Huazhong University of Science and Technology

Yang Li

Renmin University of China

Xu-Dong Tang 
China Academy of Chinese Medical Sciences Xiyuan Hospital

\section{Fang Lu}

China Academy of Chinese Medical Sciences

\section{Research Article}

Keywords: Zhizhu Kuanzhong, Functional dyspepsia, Postprandial distress syndrome, Randomized controlled trial

Posted Date: November 22nd, 2021

DOl: https://doi.org/10.21203/rs.3.rs-757640/v1

License: (c) (1) This work is licensed under a Creative Commons Attribution 4.0 International License. Read Full License 


\section{Abstract}

Background: Functional dyspepsia (FD) is one of the most common functional gastrointestinal disorders. Based on the various symptoms present in patients with functional dyspepsia-postprandial distress syndrome (FD-PDS), routine agents such as acid suppressants, prokinetic drugs, and centrally acting drugs, offer limited treatment choice with potential side effects. As preliminary clinical trial showed that the marketed product Zhizhu Kuanzhong Capsule (ZZKZ) can improve symptoms in FD-PDS patients, our study aims to provide further evidence on the clinical efficacy and safety of ZZKZ in the treatment of patients with FD-PDS.

Methods: In this multicenter, randomized, double-blind, placebo-controlled, parallel group clinical trial, we recruit patients with FD-PDS from 18 hospitals in China and Australia. The trial enrolls patients with FDPDS based on the Rome IV diagnostic criteria. A total of 480 eligible patients are randomized 1:1 into either ZZKZ or placebo group with 8 weeks of treatment and 4 weeks of follow-up. The primary endpoint is measured by self-rated Visual Analogue Score (VAS) for the degree of discomfort with both symptoms of postprandial fullness and early satiation, recorded once a day and 7 days a week. The primary analysis aims to compare the response rate for FD-PDS VAS score between the groups before and after 8 weeks of treatment with an alpha level of 0.05 (2-sided).

Discussion: This trial aims to strengthen the evidence for the efficacy and safety of ZZKZ, a marketed product, in treating FD-PDS. Compared to previous clinical trial targeted FD-PDS, this trial has 8-week double-blind treatment period to investigate the effect of long-term mediation through comparison with the placebo group.

Trial registration: This study is registered with ClinicalTrials.gov on 28 January 2019, number NCT03825692.

\section{Background}

Functional dyspepsia (FD) is one of the most common functional gastrointestinal disorders[1]. Patients can experience a range of dyspeptic symptoms including postprandial fullness, early satiety, midepigastric pain, and mid-epigastric burning sensation. According to the symptom pattern, FD can be divided into two subtypes: postprandial distress syndrome (PDS) and epigastric pain syndrome (EPS)[1]. FD's pathogenesis may be related to a series of factors such as gastroduodenal dyskinesia, visceral hypersensitivity, increased secretion of gastric acid, and psychological factors such as anxiety and depression[2, 3]. Based on the various symptoms present in patients with functional dyspepsiapostprandial distress syndrome (FD-PDS), routine agents such as acid suppressants, prokinetic drugs, and centrally acting drugs, offer limited treatment choice with potential side effects[4]. As the symptoms of FD are prone to recur, safe and effective medications for long-term use becomes a critical concern for the patients and clinicians $[5,6]$. 
Zhizhu Kuanzhong Capsule (ZZKZ) (National Medical Products Administration (NMPA) approval number Z20020003), manufactured by Shuangren Pharmaceutical Co., Ltd. of Lonch Group, has been marketed for more than 10 years in China. ZZKZ is originated from the ancient document "Synopsis of Prescriptions of the Golden Chamber" which mainly composed of 4 kinds of Chinese herbs: Rhizoma Atractylodis Macrocephalae, Fructus Aurantii Immaturus, Radix Bupleuri, and Fructus Crataegi. These herbs have been long applied in traditional Chinese Medicine (TCM) for alleviating gastrointestinal symptoms. In a multicenter clinical trial in China which applied Cisapride as control, 403 cases (105 cases in the control group, 196 cases in the treatment group, and 102 cases in the open group) were investigated[7]. The results showed that ZZKZ alone could relief FD symptoms with an overall response rate of up to $89.2 \%$. In a double-blind, randomized, placebo-controlled study of 392 subjects in 2017, ZZKZ was found to significantly improve symptoms such as early satiety and postprandial fullness discomfort in patients with FD-PDS[8].

ZZKZ also exhibited therapeutic effects on anxiety and depression through both animal experiments and clinical studies. An animal study showed that ZZKZ improved the depressive behaviors of the animals, which the mechanism may be associated with the improvement of 5-HT neuron transmission and the inhibition of stress-induced corticoid secretion[9]. A multicenter clinical study of 202 patients showed that ZZKZ reduced the depression score in patients with FD-PDS and improved the depressive symptoms[10]. Another study showed that in patients with FD accompanied with anxiety and depression, there was no significant difference between the 4-week ZZKZ group and Domperidone plus Neurostan group in the improvement of anxiety and depression scores[11].

In this trial, placebo is selected as the control since placebo design makes the trial easier for researchers to assess the side effects of ZZKZ and reduce biases through comparison of the groups. With evidence from the preliminary clinical trial investigated the short-term use of ZZKZ[12], further well-designed clinical trial should be carried out to consolidate the short-term evidence and explore the effect for longterm use of the medication. Therefore, we designed this trial to evaluate the clinical efficacy and safety of ZZKZ in the treatment of FD-PDS.

\section{Methods And Analysis}

\section{Trial design}

This multicenter, randomized, double-blind, placebo-controlled, parallel group study is conducted at 18 hospitals in China and Australia. Patients fulfilling the Rome IV FD-PDS criteria[13] are recruited primarily through advertisements on hospital websites and outpatient clinics. Before randomization, all patients are required to provide written informed consent. In response to the COVID-19 pandemic, face-to-face site visits may be replaced by telephone consultations, and study materials may be distributed and returned via mail. The study protocol (version XYYY-V-2.1) has been approved by ethics committees at all participating hospitals following the Declaration of Helsinki and is reported based on SPIRIT 
(Supplementary file 1)[14]. This trial has been registered on ClinicalTrials.gov (NCT03825692). A flow diagram of the trial is shown in Fig. 1.

\section{Eligibility criteria}

Inclusion criteria

(1) Outpatients aged 18-65 years; (2) meeting the Rome IV diagnostic criteria for FD-PDS; (3) able to discontinue prohibited medications that may affect the evaluation of the effectiveness; (4) with Visual Analogue Score (VAS) score $\geq 4$ for major symptoms (at least one of postprandial fullness discomfort and early satiety); and (5) informed and voluntarily sign the informed consent form.

\section{Exclusion criteria}

(1) Gastroscopic findings of gastric cancer, peptic ulcer, erosive gastritis (grade 2 or higher), moderate to severe atrophic gastritis, dysplasia, or other organs gastrointestinal disease; (2) history of abdominal surgery (except for appendectomy and cesarean section); (3) defects in immune function, or using immunosuppressive agents or glucocorticoids within the past 3 months; (4) combined severe heart and lung insufficiency, liver and kidney dysfunction, an endocrine disorder, hematopoietic disorder, hematological tests revealed iron deficiency anemia; (5) severe anxiety or depression; (6) psychotic patients and those with intellectual or language disabilities; (7) pregnancy or breastfeeding; (8) allergy to the ingredients of the investigational drug; (9) drug or alcohol abuse; (10) participated in clinical trials in the past 3 months; (11) been regarded by the investigator as not feasible for this clinical trial.

\section{Randomization and allocation concealment}

Stratified-block randomization method by SAS 9.3 statistical software was used to centrally generate a random number table for the patients according to the number of cases allocated to each participating hospital with a ratio of 1:1 and performed by a statistician in a third institution. Parameters of the selected block's length and the random initial seed are sealed together as secret data in the blind codes. Each drug is randomly coded with an identification number according to the random number table and distributed to each site. Each site further dispenses the drug identification numbers according to the order of the subject's visit and cannot alter the numbering arbitrarily.

\section{Blinding}

Both patients and investigators are blinded to the assigned group. To achieve the blind method, the blind codes of this study which consist of two levels, are held by the sponsor. The level-I blind code designates whether each drug number is associated with drug A or drug B, respectively; the level-II blind code designates whether drug $A$ and drug $B$ is a trial drug or a placebo. According to the statistical analysis plan, the level-I blind code will not be revealed until the statistical analysis is started, which a statistical analyst will analyze. After the analyst writes a report, we will hold a concluding meeting, the level-II blind code is revealed for the first time on the spot, and the unblinded staff sign on the blind code. 
Unblinding is permissible when serious adverse events occur and the actions to protect the safety of the subject must be taken immediately during the study. When urgent unblinding is required, the treating physician shall report to the principal investigator of the site. After approval, the emergency letter sent with the drug will be unpacked, the type of drug administered will be identified, a prompt rescue will be made, and the potential reasons will be clearly explained, the medical record will be signed and dated. Concurrently, the subject will be discontinued from the trial.

\section{Interventions}

Experimental: ZZKZ (0.43 g per capsule) (Lonch Group Shuangren Pharmaceutical Co., Ltd.; batch number: Z20020003) is composed of the following TCM crude drugs: Rhizoma Atractylodis Macrocephalae $(0.16 \mathrm{~g}$, accounting for $37.5 \%)$, Fructus Aurantii Immaturus $(0.11 \mathrm{~g}$, accounting for $25 \%)$, Radix Bupleuri $(0.08 \mathrm{~g}$, accounting for $18.75 \%)$, and Fructus Crataegi $(0.08 \mathrm{~g}$, accounting for $18.75 \%)$. The parts of plant used are listed in Chinese Pharmacopoeia (version 2015). ZZKZ is a standardized product that used the four dry herbs and manufactured as concentrated granules (using $65 \%$ ethanol as solvent), and packed into capsules. The ratio of herbal drug to extract is $2.79: 1$. The lot numbers of the raw materials are kept by the manufacturer with the authentication of raw materials. Qualitative tests include chromatographic analysis, microscopic identification, thin-layer identification, examinations of moisture, impurities, total ash, heavy metals and harmful elements, and residual sulfur dioxide, were carried out for ZZKZ and all the four herbs by the manufacturer.

Placebo: ZZKZ mimics (0.43 g per capsule) (Lonch Group Shuangren Pharmaceutical Co., Ltd.). The placebo does not contain any drug, and starch and microcrystalline cellulose are used as filling agents. The placebo for ZZKZ capsules $(0.43 \mathrm{~g})$ contain starch and microcrystalline cellulose in a ratio of 2:1, with additional amount of an appropriate amount of a food-grade pigment solution, using magnesium stearate as a lubricant ( $0.5 \%$ ). Food additives that meet China's quality standards for pharmaceutical excipients are used for placebo color modulation. The placebo has the same taste, odor, and color as ZZKZ. Dose, method of use, and duration of the investigational products are listed in Table 1.

Table 1

Dose and duration of the investigational products

\begin{tabular}{|llll|}
\hline Group & $\begin{array}{l}\text { Investigational } \\
\text { Product }\end{array}$ & Dose and Methods & $\begin{array}{c}\text { Duration } \\
\text { (weeks) }\end{array}$ \\
\hline ZZKZ & ZZKZ & $\begin{array}{l}3 \text { capsules at a time, 3 times a day, taken orally 10-15 } \\
\text { min before meals }\end{array}$ & 8 \\
\hline Placebo & ZZKZ Mimics & $\begin{array}{l}3 \text { capsules at a time, 3 times a day, taken orally 10-15 } \\
\text { min before meals }\end{array}$ & 8 \\
\hline
\end{tabular}

Concomitant drug

Long-term concomitant medication which may have treatment effect on FD should be avoided: 
(1) Medicines related to the treatment of FD should not be administered during the study.

(2) Patients taking non-steroidal anti-inflammatory drugs such as aspirin before enrollment may continue taking these drugs without increasing or reducing the dose.

(3) Opioid preparations are prohibited during the trial. If used before enrollment, sedative and hypnotic drugs (e.g., patients with insomnia take diazepam, etc. as needed) and antidepressants can be continued without increasing or decreasing the dose.

(4) Some antibiotics that affect gastric motility are prohibited during the trial: macrolide antimicrobial or azole antifungal.

(5) During the trial, acupuncture, massage, cupping, and other TCM therapies related to the treatment of this disease shall not be used.

(6) If the participant is eligible by the inclusion criteria but needs to continue taking medicine or to add other drugs or treatment methods for the consideration of comorbid illness or changes of illness state, it is necessary to record all information to the case report form, including the name of the drug (or other therapies), the dosage, use frequency and time.

\section{Outcomes}

\section{Primary outcome}

Participants self-rate on the VAS for the degree of discomfort with both symptoms of postprandial fullness and early satiation, Degree of discomfort is indicated by a 10-cm line marked from 0 (Asymptomatic or No Discomfort) to 10 (Extreme Severe or Extreme Discomfort)[15]. The rating is made once a day and 7 days a week via a diary card. For VAS scores for postprandial fullness discomfort and early satiety, the integral average for both symptoms over the past week is evaluated, and a $50 \%$ decrease from baseline in the integral average at 8 weeks is recorded as a response. The proportion of the response at 8 weeks after randomization is considered the primary efficacy endpoint.

\section{Secondary outcomes}

\section{VAS score of each FD symptom}

On the diary cards, subjects recorded the VAS of each FD symptom daily, including abdominal distension (mid-epigastric distension or lower abdominal distension), abdominal pain (mid-epigastric pain or lower abdominal pain), epigastric burning sensation, nausea, excessive eructation, heartburn, vomiting, regurgitation, dysphagia, abdominal enlargement, and defecation smoothness[16]. VAS is scored on a scale of $0-10$ which the higher the score, the severer the sign is. The record is made once a day and 7 days a week. The investigators use the average VAS scores as the symptom intensity score for the week. The change in each symptom's score at 8 weeks after randomization relative to the baseline is evaluated. 


\section{Overall treatment response rate}

The overall treatment efficacy is evaluated using a 7-point Likert Overall Evaluation Scale[17]. The clinical investigators will ask the subjects the following question weekly: "In the last week, how much have your dyspeptic symptoms been alleviated as compared to pre-treatment?" There are 7 options: (1) the symptoms improved significantly, (2) the symptoms improved, (3) the symptoms improved slightly, (4) the symptoms did not change, (5) the symptoms aggravated somewhat, (6) the symptoms aggravated, (7) the symptoms aggravated significantly. At the last visit time point of the treatment cycle, patients who selected (1) and (2) are defined as treatment responders, and those who chose (3) to (7) are defined as non-responders. The response rates at 8 weeks after randomization between the groups are compared for differences.

\section{Short Form Nepean Dyspepsia Index (SFNDI)}

SFNDI is a reliable and valid measure of the quality of life in functional dyspepsia with 10 items on how subjects' stomach pain, discomfort, or other epigastric symptoms over the last 14 days affected their lives[17]. Add up the 10 items for each of the five subscale scores (range of each sub-scale 2-10). The SFNDI score changes at 4 weeks and 8 weeks after randomization relative to the baseline are calculated.

\section{Hospital Anxiety and Depression Scale (HAD) score}

$H A D$, mainly used in patients in general hospitals, provides two sets of tests to assess anxiety and depression respectively[18]. Among them, " $A$ " stands for anxiety items, " $D$ " stands for depression items, and each item is scored at four levels. Each of the two sets of items is superimposed to obtain their respective total score. A total score of 0 to 7 indicates normal, 8 to 10 indicates borderline abnormal, and 11 to 21 indicates abnormal. The HAD score changes at 4 weeks and 8 weeks after randomization relative to the baseline are calculated.

\section{Safety observation}

1. Vital signs: Body temperature, heart rate, blood pressure, and respiration (once at each follow-up visit).

2. Blood routine, urine routine, stool routine plus fecal occult blood test (once before and after treatment respectively, twice in total).

3. Hepatic function (alanine aminotransferase (ALT), aspartate aminotransferase (AST), total bilirubin (TBil), alkaline phosphatase (ALP), gamma-glutamyl transferase (GGT)), renal function (serum creatinine $(\mathrm{SCr})$, blood urea nitrogen (BUN)) (once before and after treatment respectively, twice in total).

4. Electrocardiogram (once before and after treatment respectively, twice in total).

5. Severity and incidence of adverse events (recorded in detail at any time).

During the trial, each participant receives safety monitoring. Potential adverse effects are identified from the data collected by the post-marketing adverse drug reaction monitoring center, and the safety data from preliminary trials[19]. All adverse events will be reported to the ethics committee and office of clinical trials institution. They review all documented harms during the trial and make judgement with 
regards to causality. The schedule for the enrollment, interventions, and assessment is summarized in Fig. 2.

\section{Data management}

There are two ways for patients to record their diary cards. using either paper diary cards or an electronic diary (eDiary). Both paper diary cards and eDiary record the severity of patients' symptoms related to FD, including postprandial fullness discomfort, early satiety, abdominal distension (mid-epigastric distension or lower abdominal distension), abdominal pain (mid-epigastric pain or lower abdominal pain), epigastric burning sensation, nausea, excessive eructation, heartburn, vomiting, regurgitation, dysphagia, abdominal enlargement, and defecation smoothness. All the investigators receive training regarding data management and participants' education for instructions on using the paper diary cards and/or eDiary. For eDiary, in order to ensure that participants can fill out the diary card every day, the system sends a text message to the participants who have not filled out the diary card every night for reminder. Also, researchers can use the system to check whether participants have filled out the diary card. The original medical records are kept intact by the site investigators as original documents. The data are inputted into the eCRF which the database has been established before recruitment. Investigators are responsible for verifying the accuracy of the data. Data locking is completed by the data management team, researchers cannot modify the data after locked. All research data, including paper and electronic documents, will be kept for at least 5 years after publication. All participant data collected during the trial will be deidentified and available for anyone who wishes to access the data immediately following publication.

A Data and Safety Monitoring Board (DSMB) is set up to review the protocol and the research data. The DSMB members meet regularly to review the trial data according to ethical and safety standards, and monitor the data's authenticity and completeness based on the study design. The DSMB also review the trial progress, determine adverse events, and have the authority to decide whether the study needs to end early.

\section{Quality control and assurance}

This trial protocol is reviewed and revised by experts in gastroenterology, methodology, and statistics. Investigators have been trained with a prespecified standard operating procedure before the trial, including eligibility criteria, interventions, details in filling eCRF, assessment of outcomes, and data management. An inspection plan is designed for quality control and professionals are hired to supervise the research process. Moreover, patients are allowed to use authorized accounts in WeChat to fill out the eCRF to improve compliance. The system will send reminders to patients for filling out the eCRF. In addition, the research assistants and clinical research coordinators can monitor participants' research records through the researcher management backstage.

\section{Sample size calculation}

The study's treatment duration is 8 weeks, with the responder rate of the clinical symptoms of FD-PDS after 8 weeks of treatment being the primary efficacy endpoint. To control the overall false-positive error 
in the trial and to ensure the power of the test, the study adopts the following parameters: alpha level of 0.05 (2-sided), power of 0.8 , with the ratio of number of cases in the ZZKZ group and the placebo group as 1:1. According to the results of a multi-center clinical study of ZZKZ completed by Professor Chen Minhu in the First Affiliated Hospital of Sun Yat-sen University, with the cooperation of 16 domestic hospitals, the responder rate for clinical symptoms of FD-PDS was $54 \%$ with the ZZKZ and $38 \%$ for the placebo[12]. Calculation of sample size indicates that 187 cases are required in the ZZKZ and placebo groups respectively. Assuming dropout rate of $20 \%$, a total of 480 cases is required for our study.

\section{Statistical analysis}

Statistical analysis will be performed using SAS 9.3 software. All statistical tests will use a two-sided test, $P \leq 0.05$ will be regarded as significant. A $95 \%$ confidence interval will be used. Based on the intention-totreat principle, the missing data will be replaced by the last data (i.e., the missing efficacy data will be replaced by the efficacy data of the previous follow-up). The efficacy analysis will be mainly based on a full analysis set, and the central effect will be considered. The primary efficacy endpoint will be tested for superiority. The efficacy between groups will be compared using the Cochran-Mantel-Haenszel- $\chi 2$ test with and without regard to center stratification, and a $95 \%$ confidence interval of the difference between groups will be calculated. Sub-analysis will be carried out to review whether there is a difference in the effect of ZZKZ according to demographical data across countries. The safety analysis will be mainly based on descriptive statistics. The adverse events that occur in this trial will be described in a list, and the incidences will be compared using Fisher's exact probability test.

\section{Ethics and dissemination}

Institutional ethics approvals for the trial have been obtained from the ethics committee of Xiyuan Hospital and all other participating institutions (Supplementary file 2). The purpose and potential risks of this study are fully explained to the participants. All participants are required to provide written informed consent before participating in this study. Model consent form is attached (Supplementary file 3).

Practitioners in gastroenterology who have been authorized as investigators will obtain the consent of potential trial participants. The investigators will explain the study procedures to the potential participants in the language and text that they can understand. After the potential participants have thoroughly read and understood the informed consent form, the informed consent form will be signed and dated by the patient if they agree to participate.

After the patient has signed the informed consent form, relevant personal information will be collected and a subject screening number will be assigned. All information about the patient, including his/her identity, medical history, and illness, will be kept strictly confidential by the researcher. Access to the records is restricted to authorized investigators and ethics committees only. In all the documents submitted to the sponsor, only the subject screening number for the clinical study can be used to identify the clinical study subject, without indicating the subject's name and hospital reference number. The subjects' names will not appear in any public information or reports related to the study. 
In case it is necessary to modify the study protocol after the trial has begun, the principal investigator and sponsor will reach a consensus and resubmit the modified protocol to the ethics committee. After obtaining the approval, the modified protocol shall be uploaded to the trial registry and submitted to the researchers in other sub-centers for approval by the ethics committees. Only with the support of local ethics committee can further research be carried out. Patients participating in the study will be informed if their benefits are involved.

The final study dataset will be submitted to the National Data Center for Population and Health Sciences after completion of the project, and may be available for investigators upon reasonable request.

\section{Discussion}

FD-PDS is prone to recur which seriously affects patients' quality of life, and brings a huge demand for medical resources[20]. Complied with good clinical practice guideline and had been examined with clinical trials[19], our study assesses and compares the effects of ZZKZ with placebo for treatment of FDPDS in China and Australia as an attempt to observe the effects across different populations. Compared to previous clinical trial targeted FD-PDS [12], we set up an 8-week double-blind treatment period to investigate the effect of long-term mediation through comparison with the placebo group[21, 22].

We focus on the patient-reported outcomes to capture the patients' illness experience in a structured format as a reflection of the real-world clinical situation[23]. The use of a daily diary has the advantages of consecutive recording of disease activity and allowing average values by repeated measurements. Evaluation of the VAS of each FD symptom through daily measurement is considered the most reliable tool to evaluate symptom pattern and severity, however, filling out diary cards daily is a big challenge for maintaining high compliance of the participants.

FD-PDS is associated with mental stress. Previous animal studies have shown that ZZKZ has antidepressant effect that can relief depressant behaviors[9]. To investigate the effect in patients with FDPDS, HAD is adopted in this clinical trial to observe psychological changes of the participants. Moreover, since studies have shown that symptoms may have an inverse relationship with the severity of H. pyloriassociated inflammation and oxidative damage in patients with FD[24, 25], further studies can consider to investigate the effect of ZZKZ in Helicobacter pylori-positive patients. TCM has unique theoretical and practical methods in treating diseases, and treatment based on syndrome differentiation is the basic principle of disease recognition and treatment in TCM[26]. As medical records, TCM symptoms (gastrointestinal symptoms, mood, urine, feces, tongue texture, tongue coating, etc.) of the participants are collected in this study.

There were some limitations in this study. Firstly, ZZKZ is composed of 4 TCM ingredients, and its complex treatment mechanisms requires further investigation. Secondly, since this study is conducted in two countries mainly among the Chinese populations, it limits the generalizability of the results. Thirdly, because of the COVID-19 pandemic, some face-to-face site visits are replaced by telephone consultations, which may create challenges for the collection of data. Lastly, although biomarker provides supporting 
evidence for the clinical efficacy of FGID treatment, we do not use biomarkers to evaluate the efficacy of the drugs in this study, which lead to research gap in the explanation of the clinical results from the aspect of biological indicators[22].

\section{Abbreviations}

FD: functional dyspepsia; ZZKZ: Zhizhu Kuanzhong Capsule; PDS: postprandial distress syndrome; EPS: epigastric pain syndrome; NMPA: National Medical Products Administration; TCM: traditional Chinese Medicine; VAS: Visual Analogue Score; SFNDI: Short Form Nepean Dyspepsia Index; HAD: Hospital Anxiety and Depression Scale; ALT: alanine aminotransferase; AST: aspartate aminotransferase; TBil: total bilirubin; ALP: alkaline phosphatase; GGT: gamma-glutamyl transferase; SCr: serum creatinine; BUN: blood urea nitrogen; eDiary: electronic diary; DSMB: Data and Safety Monitoring Board.

\section{Trial Status}

The trial has been recruiting patients since Oct 24,2019 . The recruitment will be completed before December 2022.

\section{Declarations}

\section{Ethics approval and consent to participate}

Ethics committees have approved the study protocol (version XYYY-V-2.1) at all participating hospitals following the Declaration of Helsinki. Signed informed consent form is required for every patient to participant in the trial.

\section{Patient consent for publication}

Not applicable.

\section{Availability of data and materials}

All individual participant data collected during the trial will be deidentified and available for anyone who wishes to access the data immediately following publication.

\section{Competing interests}

The authors declare that they have no competing interests.

\section{Funding}

The study is supported by Special Research on Modernization of Traditional Chinese Medicine in the National Key Research and Development Program in China's 13th Five-Year Plan Demonstrative Research on TCM-related International Cooperation with Countries along the Belt and Road (No.2017YFC1703703). 
The funding plays no role in study design, collection, management, analysis, interpretation of data, writing of the report, or the decision to submit the report for publication.

\section{Author contributions}

FL and XDT designed the study. FL drafted the protocol. MX, LLDZ, and WCL drafted the manuscript. All authors made significant contributions to the conception and design of the study protocol. YZ and LLDZ assisted with the development of the study design and protocol. The protocol has been reviewed by KAG, $\mathrm{GH}, \mathrm{JT}, \mathrm{HS}, \mathrm{MHC}, \mathrm{XH}, \mathrm{YX}, \mathrm{JL}$. YL wrote the statistical analysis plan. All authors gave final approval of the manuscript and agreed to be accountable for all aspects of the work.

\section{Acknowledgments}

We are thankful to the participants for their participation.

\section{References}

1. Stanghellini V. Functional Dyspepsia and Irritable Bowel Syndrome: Beyond Rome IV. Dig Dis. 2017;35(Suppl 1):14-7.

2. Walker MM, Potter MD, Talley NJ. Tangible pathologies in functional dyspepsia. Best Practice \& Research Clinical Gastroenterology. 2019;40-41:101650.

3. Van Oudenhove L, Aziz Q. The role of psychosocial factors and psychiatric disorders in functional dyspepsia. Nat Rev Gastroenterol Hepatol. 2013;10(3):158-67.

4. Corsetti M, Fox M. The management of functional dyspepsia in clinical practice: what lessons can be learnt from recent literature? F1000Res. 2017;6:1778.

5. Masuy I, Van Oudenhove L, Tack J. Review article: treatment options for functional dyspepsia. Aliment Pharmacol Ther. 2019;49(9):1134-72.

6. Vijayvargiya P, Camilleri M, Chedid V, Mandawat A, Erwin PJ, Murad MH. Effects of Promotility Agents on Gastric Emptying and Symptoms: A Systematic Review and Meta-analysis. Gastroenterology. 2019;156(6):1650-60.

7. Xu C, Xu D, Kou Q, Zhang J, Feng W. Evaluation of Zhizhukuanzhong capsule in treatment of functional dyspepsia in 403 patients: a phase II clinical trial. Chinese Journal of New Drugs Clinical Remedies. 2004;23(8):493-7.

8. Li W, Wen H, Song Q. Effects of Atractylodes macrocephala extracts and its components on the activities of salivary amylase. Lishizhen Medicine Materia Medica Research. 2006;17(11):2123-5.

9. Chang H, Liang J, Shi R, Cheng K, Sun W. Antidepressant effect of Zhizhukuanzhong Capsules on animal model of depression. Journal of Beijing University of Traditional Chinese Medicine. 2009;32(10):690-4.

10. Guan S, Li Y. Clinical study of Zhizhu Kuanzhong capsule in treatment of patients with functional dyspepsia complicated with anxiety and depression. Practical Pharmacy Clinical Remedies. 
2016;19(3):296-9.

11. Chassany O, Marquis P, Scherrer B, Read NW, Finger T, Bergmann JF, Fraitag B, Geneve J, Caulin C. Validation of a specific quality of life questionnaire for functional digestive disorders. Gut. 1999;44(4):527-33.

12. Xiao Y, Li Y, Shu J, Li Y, Xu J, Ren J, Liu D, Wang J, Zhou L, Li Y, Tang G, Tian D, Zhang S, Hou X, Wang H, Li Z, Lv N, Chen M. The efficacy of oral Zhizhu Kuanzhong, a traditional Chinese medicine, in patients with postprandial distress syndrome. J Gastroenterol Hepatol. 2019;34(3):526-31.

13. Talley NJ, Walker MM, Holtmann G. Functional dyspepsia. Curr Opin Gastroenterol. 2016;32(6):46773.

14. Chan AW, Tetzlaff JM, Gøtzsche PC, Altman DG, Mann H, Berlin J, Dickersin K, Hróbjartsson A, Schulz KF, Parulekar WR, Krleža-Jerić K, Laupacis A, Moher D. SPIRIT 2013 Explanation and Elaboration: Guidance for protocols of clinical trials. BMJ. 2013;346:e7586.

15. Park JW, Ryu B, Yeo I, Jerng UM, Han G, Oh S, Lee J, Kim J. Banha-sasim-tang as an herbal formula for the treatment of functional dyspepsia: a randomized, doubleblind, placebo-controlled, two-center trial. Trials. 2010;11:83.

16. Kim S, Hilli L, Orlowski L, Kupperman J, Baral JL, Waters MF. R. Efficacy of Probiotics and Nutrients in Functional Gastrointestinal Disorders: A Preliminary Clinical Trial. Dig Dis Sci. 2006;51(12):213444.

17. Porika SK, Veligandla KC, Muni SK, Acharya S, Mehta SC, Sharma AD. RealWorld. Non-Interventional, Observational Study to Evaluate Effectiveness and Tolerability of Acotiamide Hydrochloride Hydrate in Treatment of Functional Dyspepsia. Adv Ther. 2018;35(11):1884-93.

18. Sin SH, Wu J, Kang Y, Yip KHK, Kong NS, Wan H, Ng BFL, Chen H. Efficacy of modified Banxia Xiexin decoction in the management of Wei-Pi syndrome (postprandial distress syndrome): study protocol for a randomized, waitlist-controlled trial. Trials. 2021;22(1):135.

19. Wen MY, Zhang FC, Wang YJ. Effect of Zhizhu Kuanzhong Capsules on Treatment of Functional Dyspepsia: A Meta-Analysis of Randomized Controlled Trials. Chin J Integr Med. 2019;25(8):625-30.

20. Halling K, Kulich K, Carlsson J, Wiklund I. An international comparison of the burden of illness in patients with dyspepsia. Dig Dis. 2008;26(3):264-73.

21. Khanna P, Agarwal N, Khanna D, Hays RD, Chang L, Bolus R, Melmed G, Whitman CB, Kaplan RM, Ogawa R, Snyder B, Spiegel BM. Development of an online library of patient reported outcome measures in gastroenterology: the GI-PRO database. Am J Gastroenterol. 2014;109(2):234-48.

22. Enck P, Klosterhalfen S. Placebo Responses and Placebo Effects in Functional Gastrointestinal Disorders. Front Psychiatry. 2020;11:797.

23. Basch E. Patient-Reported Outcomes - Harnessing Patients' Voices to Improve Clinical Care. N Engl J Med. 2017;376(2):105-8.

24. Suzuki H, Hibi T, Marshall BJ. Helicobacter pylori: present status and future prospects in Japan. J Gastroenterol. 2007;42(1):1-15. 
25. Turkkan E, Uslan I, Acarturk G, Topak N, Kahraman A, Dilek FH, Akcan Y, Karaman O, Colbay M, Yuksel S. Does Helicobacter pylori-induced inflammation of gastric mucosa determine the severity of symptoms in functional dyspepsia? J Gastroenterol. 2009;44(1):66-70.

26. Jiang M, Lu C, Zhang C, et al. Syndrome differentiation in modern research of traditional Chinese medicine. J Ethnopharmacol. 2012;140(3):634-42.

Figures

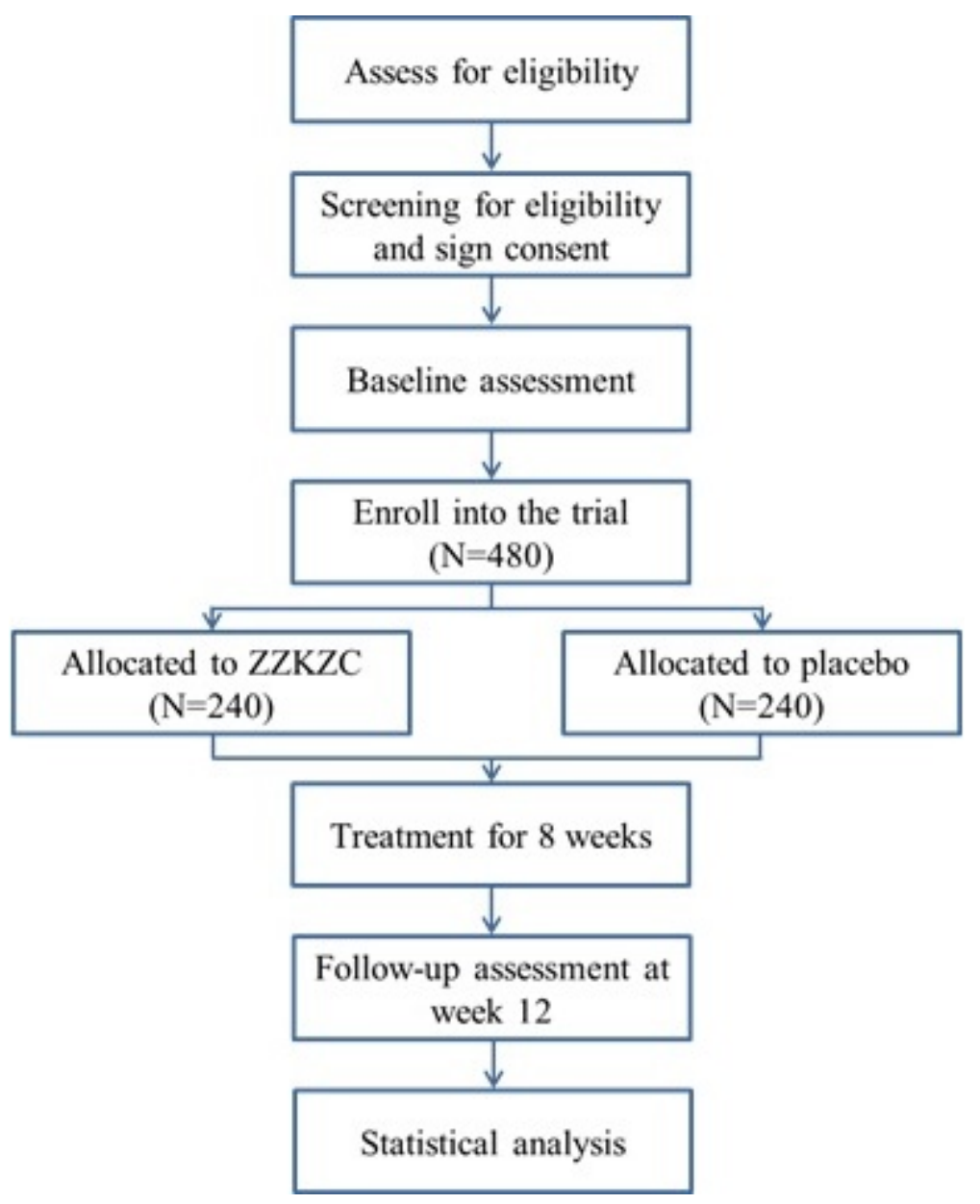

Figure 1

Flow diagram of the trial 


\begin{tabular}{|l|c|c|c|c|c|c|c|c|}
\hline & \multicolumn{7}{|c|}{ STUDY PERIOD } \\
\hline \multicolumn{1}{|c|}{$\begin{array}{c}\text { Time Point } \\
\text { (week) }\end{array}$} & $\begin{array}{c}\text { Washout } \\
(-3 \text { to }-1)\end{array}$ & $\begin{array}{c}\text { Run-in } \\
(-1)\end{array}$ & 0 & 2 & 4 & 6 & 8 & 12 \\
\hline Enrollment & & & & & & & & \\
\hline $\begin{array}{l}\text { Eligibility } \\
\text { screen }\end{array}$ & $\mathrm{x}$ & & & & & & & \\
\hline $\begin{array}{l}\text { Informed } \\
\text { consent }\end{array}$ & $\mathrm{x}$ & & & & & & & \\
\hline Randomization & & & $\mathrm{x}$ & & & & & \\
\hline Allocation & & & $\mathrm{x}$ & & & & & \\
\hline Intervention & & & & & & & & \\
\hline ZZKZ & & & & $\mathrm{x}$ & $\mathrm{x}$ & $\mathrm{x}$ & $\mathrm{x}$ & \\
\hline Placebo & & & & $\mathrm{x}$ & $\mathrm{x}$ & $\mathrm{x}$ & $\mathrm{x}$ & \\
\hline Assessment & & & & & & & & \\
\hline VAS & & $\mathrm{x}$ & $\mathrm{x}$ & $\mathrm{x}$ & $\mathrm{x}$ & $\mathrm{x}$ & $\mathrm{x}$ & $\mathrm{x}$ \\
\hline OTE & & & & $\mathrm{x}$ & $\mathrm{x}$ & $\mathrm{x}$ & $\mathrm{x}$ & $\mathrm{x}$ \\
\hline HAD & & & $\mathrm{x}$ & & $\mathrm{x}$ & & $\mathrm{x}$ & $\mathrm{x}$ \\
\hline SFNDI & & & $\mathrm{x}$ & & $\mathrm{x}$ & & $\mathrm{x}$ & $\mathrm{x}$ \\
\hline Safety & & & & & $\mathrm{x}$ & $\mathrm{x}$ & $\mathrm{x}$ & $\mathrm{x}$ \\
\hline
\end{tabular}

Figure 2

Schedule of the trial

\section{Supplementary Files}

This is a list of supplementary files associated with this preprint. Click to download.

- Supplementaryfile1SPIRITchecklist20210726.pdf

- Supplementaryfile2Modelconsentform.pdf 